\title{
New Method for Fourier Coefficient Estimation from Irregular Samples
}

\author{
Predrag B. Petrović
}

\begin{abstract}
The paper deals with the estimation of amplitude and phase of an analogue multi-harmonic band-limited signal from irregularly spaced sampling values. To this end, assuming the signal fundamental frequency is known in advance (i.e., estimated at an independent stage), a complexity-reduced algorithm for signal reconstruction in time domain is proposed. The proposed algorithm for the calculation of the unknown parameters requires $O\left((2 M+1)^{2}\right)$ flops, while the straightforward solution of the obtained equations takes $O\left((2 M+1)^{3}\right)$ flops $(M$ is the number of the harmonic components). The paper investigates the errors related to the signal parameter estimation, and there is a computer simulation that demonstrates the accuracy of these algorithms.
\end{abstract}

Index Terms-Band-limited signals; fourier coefficient estimation; analytical solutions; signal reconstruction; time.

\section{INTRODUCTION}

Estimating the amplitude and phase of a signal accurately is very important in many areas, even when the frequencies contained in the signal are already known [1]. These include the dual-tone multiple frequencies (DTMF) signal detection in digital communications, ECG sinusoidal interference cancellation, recovery of biomedical signals, and pitch detection in automated transcription. In power systems, estimation of the harmonic components is necessary to ensure the quality of power supply.

In signal processing, reconstruction usually means determination of an original continuous signal from a sequence of equally spaced samples. It is a well-known fact that any real signal which is transmitted along a channel-like form will have a finite bandwidth. As the result, the received signal's spectrum cannot contain any frequencies above a maximum value, $f_{\max }=M f$ ( $f$ is the fundamental frequency). Consequently, $M$ frequencies provide a specification of everything we know about the signal spectrum in terms of a DC level plus the amplitudes and phases of just - i.e. all the information we have about the spectrum can be specified by $2 M+1$ numbers. Many attempts have been made in respect to application of sampling techniques supported by optimal methods of reconstruction of band-limited signals in the form of a Fourier series (trigonometric polynomials) [2]-[5].

This paper analyses the sampling of an analogue multi-harmonic input signal, as opposed to the method based

Manuscript received August 30, 2011; revised December 31, 2011. This work was supported in part by the Ministry of Education and Science of the Republic of Serbia under Grants 42009 and OI-172057.

P. B Petrović is with the Technical Faculty Čačak, 32000 Čačak, Serbia (phone: +381-32-302-748; fax: +381-32-342-101; (e-mail: predragp@ tfc.kg.ac.rs) on the integration of the input analogue signal [6], along with the problem of subsequent reconstruction of the processed signals. It was noticed in [7] that the ac signal integration method produces a regular form of system matrix in the derived system of equations, provided an adequate choice is made regarding the time parameters within which the integration is done; this makes it possible to have much more efficient reconstruction procedure subsequently. If the samples were assumed to be measured without errors, the presented algorithm, without any further modifications, can be used for signal reconstruction of periodic band-limited signals, which is the situation that occurs in a simulation. In a real environment and when the measuring is performed in practice, the samples are measured with error. In this case, the suggested algorithm must be modified, in order to be able to determine the best signal estimate, according to the criterion assumed, just like in [5], [6], [8].

The proposed algorithm for estimation is based on the use of the values that were obtained as a result of the sampling of the continual input signal. This kind of processing must be done as many times as was needed to enable the determination of the unknown signal parameters. The obtained system of the linear equations can be simply solved by using the derived analytical and summarized expressions. For the estimation of amplitude and phase of a complex ac signal, the algorithm requires $\mathrm{O}\left((2 M+1)^{2}\right)$ flops, while the straightforward solution of the obtained equations takes $\mathrm{O}\left((2 M+1)^{3}\right)$ flops. For this reason, the proposed method offers a significant improvement in computational efficiency over the standard reconstruction algorithms, at a lower numerical error. The method is designed for very accurate RMS measurements of periodic signals, and can be applied for precise measurements of other important quantities such as power and energy. They can also be applied in signal reconstruction, spectral estimation, system identification, as well as in other important signal processing problems.

\section{II.PROBLEM FORMULATION}

Let us assume that the input signal of the fundamental frequency $f$ is band limited to the first $M$ harmonic components. This form of continuous signal with a complex harmonic content can be represented as a sum of the Fourier components as follows:

$$
s(t)=a_{0}+\sum_{k=1}^{M} a_{k} \sin \left(k 2 \pi f t+\psi_{k}\right)
$$

By sampling the signal (1), and by forming a system of equations of the same form, in order to determine the $2 M+1$ unknown (amplitudes and phases of the $M$ harmonic, as well 
as the average value of the signal), we obtain:

$$
s\left(t_{l}\right)=a_{0}+\sum_{k=1}^{M} a_{k} \sin \left(k 2 \pi f t_{l}+\psi_{k}\right)
$$

where $l=1,2, \ldots, 2 M+1$. The $t_{l}$ is time moment in which the sampling of the input analogue signal is done. The $s\left(t_{l}\right)$ value represents the value of the processed signal at the moment when the sampling is performed. The obtained relation can be represented in the short form as:

$$
a_{0}+\sum_{k=1}^{M} a_{k}\left(\sin \alpha_{k, l} \cos \psi_{k}+\cos \alpha_{k, l} \sin \psi_{k}\right)=s\left(t_{l}\right)
$$

where:

$$
\alpha_{k, l}=2 k \pi t_{l}=k \alpha_{l} ; \quad(k=1,2, \ldots, M) ;(l=1,2, \ldots, 2 M+1)
$$

The $\alpha_{k, l}$ are the variables determined by the moment at which the sampling is done, as well as by the frequency of the corresponding harmonic of the input periodic signal. The system determinant for the system of $2 M+1$ unknown parameters (equation (3)), can be represented as:

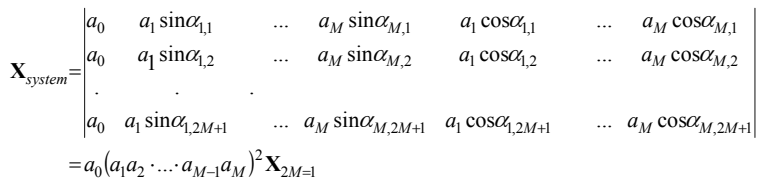

where:

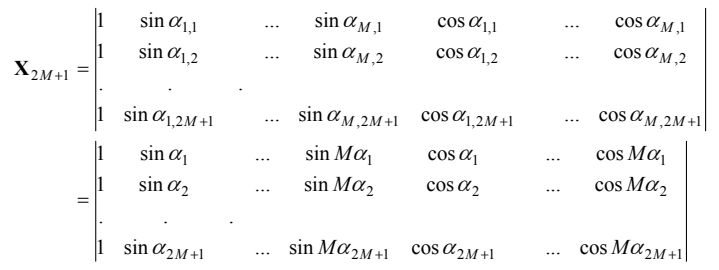

A similar form of the obtained determinant (6) was the object of study in [7]. In its form, determinant (6) resembles the well-known Van der Monde determinant. This determinant was the subject of a very intensive analysis [9-11]. However, all of these papers were proposing only the procedures that relied on program procedures (mostly iterative ones) in determining the value of the original and inverted Van der Monde's matrix with an improved efficiency. In this paper, we derive completely new analytical and summarized expressions for exactly solving the system of equations (3) by using this determinant as the starting point of the analysis. Owing to relations derived in this way, it is not necessary to use the standard procedure for solving the system of equations, as suggested in [6]. This procedure, in the case of an extremely complex spectral content of a signal, would require a powerful processor and enough time for processing.

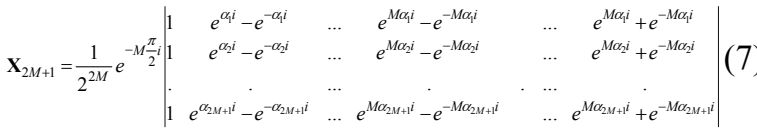

$$
\begin{aligned}
& =\left.\frac{(-1) \frac{M(M-1)}{2}}{2^{2 M}} e^{-M \frac{\pi_{i}}{2}}\right|^{-M \alpha_{i} i} e^{-(M-1) \alpha_{i} i} \quad \ldots . e^{-\alpha_{i} i} \quad 1 \quad e^{\alpha_{i} i} e^{2 \alpha_{i} i} \quad \ldots . e^{M \alpha_{i} i} \mid=
\end{aligned}
$$

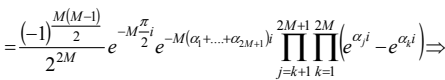

$$
\begin{aligned}
& \mathbf{X}_{2 M+1}=(-1) \frac{M(M+1)}{2} 2^{2 M^{2}} \prod_{j=k+1}^{2 M+1} \prod_{k=1}^{2 M} \sin \frac{\alpha_{j}-\alpha_{k}}{2}
\end{aligned}
$$

The given determinant (equation (6)) can be transformed in the following way (by using Euler's formulas and derivations in [7]):

The cofactors that correspond to the observed system of equations (3) are:

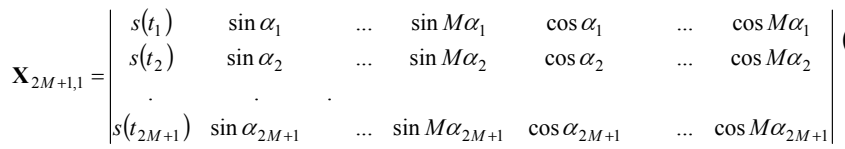

$$
\begin{aligned}
& \mathbf{X}_{2 M+1,2}=\left|\begin{array}{cccccccc}
1 & s\left(t_{1}\right) & \sin 2 \alpha_{1} & \ldots & \sin M \alpha_{1} & \cos \alpha_{1} & \ldots & \cos M \alpha_{1} \\
1 & s\left(t_{2}\right) & \sin 2 \alpha_{2} & \ldots & \sin M \alpha_{2} & \cos \alpha_{2} & \ldots & \cos M \alpha_{2} \\
\cdot & \cdot & \cdot & & & & & \\
1 & s\left(t_{2 M+1}\right) & \sin 2 \alpha_{2 M+1} & \ldots & \sin M \alpha_{2 M+1} & \cos \alpha_{2 M+1} & \ldots & \cos M \alpha_{2 M+1}
\end{array}\right|(9)
\end{aligned}
$$

And so on. The cofactors given above based on the following development can be written as:

$$
\mathbf{X}_{2 M+1,1}=s\left(t_{1}\right) \mathbf{X}_{1}^{1}+s\left(t_{2}\right) \mathbf{X}_{2}^{1}+\ldots+s\left(t_{2 M+1}\right) \mathbf{X}_{2 M+1}^{1}
$$

$\mathbf{X}_{1}^{1}, \mathbf{X}_{2}^{1}, \ldots, \mathbf{X}_{2 M+1}^{1}$ are the cofactors, obtained from cofactor $\mathbf{X}_{2 M+1,1}$ after the corresponding row as well as the first column has been eliminated. The second cofactor is derived from the expansion of $\mathbf{X}_{2 M+1}$ along such a column. For this purpose, we must determine $\mathbf{X}_{p}^{q}$ as cofactors of $\mathbf{X}_{2 M+1}$. After the intensive mathematical calculation (similar as in equation (7)) we obtain:

$$
\begin{aligned}
& \mathbf{X}_{p}^{1}=(-1)^{p+q}(-1) \frac{M(M+1)}{2} 2^{2 M(M-1)} \frac{\prod_{j=k+1}^{2 M+1} \prod_{k=1}^{2 M} \sin \frac{\alpha_{j}-\alpha_{k}}{2}}{\prod_{\substack{k=1 \\
k \neq p}}^{2 M+1} \sin \frac{\alpha_{p}-\alpha_{k}}{2}} . \\
& \cdot \sum \cos \left\{\begin{array}{l}
\frac{\alpha_{1}+\ldots+\alpha_{p-1}+\alpha_{p+1}+\ldots+\alpha_{2 M+1}}{2}- \\
-\left(\alpha_{1}+\ldots+\alpha_{p-1}+\alpha_{p+1}+\ldots+\alpha_{2 M+1}\right)_{M}
\end{array}\right\}
\end{aligned}
$$

(the summing is done by all of the M's of the set $\left.\left\{\alpha_{1}, \alpha_{2}, \ldots, \alpha_{2 M+1}\right\}\right)$.

For $2 \leq q \leq M+1$ :

$$
\begin{aligned}
& \mathbf{X}_{p}^{q}=(-1)^{p+q}(-1)^{\frac{M(M+1)}{2}} 2^{2 M(M-1)+1} \frac{\prod_{j=k+1}^{2 M+1} \prod_{k=1}^{2 M} \sin \frac{\alpha_{j}-\alpha_{k}}{2}}{\prod_{1 \leq k \neq p}^{2 M+1} \sin \frac{\alpha_{p}-\alpha_{k}}{2}} . \\
& \cdot \sum \sin \left\{\begin{array}{l}
\frac{\alpha_{1}+\ldots+\alpha_{p-1}+\alpha_{p+1}+\ldots+\alpha_{2 M+1}}{2}- \\
-\left(\alpha_{1}+\ldots+\alpha_{p-1}+\alpha_{p+1}+\ldots+\alpha_{2 M+1}\right)_{M+q-1}
\end{array}\right\}
\end{aligned}
$$

Based on (analytical) relation derived in this way the unknown parameters of the signal (amplitude, phase), can be determined through a simple division of the expression that represents a solution of the adequate co-determinants with the expression that represents an analytical solution to the system determinant: 


$$
\begin{aligned}
& q=M+r+1 \wedge 1 \leq r \leq M-1 \\
& \mathbf{X}_{p}^{q}=(-1) \frac{M(M-1)}{2} 2^{2 M(M-1)+1} \frac{\prod_{j=k+1}^{2 M+1} \prod_{k=1}^{2 M} \sin \frac{\alpha_{j}-\alpha_{k}}{2}}{\prod_{1 \leq k \neq p}^{2 M+1} \sin \frac{\alpha_{p}-\alpha_{k}}{2}} . \\
& \cdot \sum \cos \left\{\begin{array}{c}
\frac{\alpha_{1}+\ldots+\alpha_{p-1}+\alpha_{p+1}+\ldots+\alpha_{2 M+1}}{2}- \\
-\left(\alpha_{1}+\ldots+\alpha_{p-1}+\alpha_{p+1}+\ldots+\alpha_{2 M+1}\right)_{M-r}
\end{array}\right\} \\
& q=2 M+1 \\
& \mathbf{X}_{p}^{q}=(-1) \frac{M(M-1)}{2} 2^{2 M(M-1)+1} \frac{\prod_{j=k+1}^{2 M+1} \prod_{k=1}^{2 M} \sin \frac{\alpha_{j}-\alpha_{k}}{2}}{\prod_{1 \leq k \neq p}^{2 M+1} \sin \frac{\alpha_{p}-\alpha_{k}}{2}} . \\
& \cdot \cos \frac{\alpha_{1}+\ldots+\alpha_{p-1}+\alpha_{p+1}+\ldots+\alpha_{2 M+1}}{2} \\
& a_{0}=\frac{\mathbf{X}_{2 M+1,1}}{\mathbf{X}_{2 M+1}} \\
& \boldsymbol{\psi}_{k}=\frac{\operatorname{arctg} \frac{\mathbf{X}_{2 M+1, M+k+1}}{\mathbf{X}_{2 M+1, k+1}}}{A_{k} \mathbf{X}}{ }_{2 M+1} \\
& \sqrt{\mathbf{X}_{2 M+1, k+1}^{2}+\mathbf{X}_{2 M+1, M+k+1}^{2}}
\end{aligned}
$$

As shown in relation (14), the proposed processing method can be used to perform an estimation of the unknown parameters of the periodic band-limited signal, based on the explicit and summarised analytical expressions. It is a fact that the obtained system of equations (3) can be described, after the processing, by a special form of the determinant (which is summarized as the Van der Monde's determinant). This fact enables factoring and application of transformations that can be applied only on determinants. Any other procedure would lead to a much more complex calculation and to relations that are mathematically much more demanding. Owing to this, any subsequent calculation conduct towards reconstructing a periodic signal will not be related either to determinants themselves or to the procedures that are typically used in their solving. The obtained result clearly suggests that it is not necessary to use the standard procedure for solving the system of equations, as suggested in [6].

Table I presents the results obtained through the application of the derived relations for solving the observed system of equations (3), taking that $M=7, f=50 \mathrm{~Hz}, t_{l}=0.001 \mathrm{~s}$. This results were compared with the solution obtained from GEPP algorithm (Gaussian elimination with partial pivoting), offered in the Matlab program package itself (all of the calculations are done in IEEE standard double floating point arithmetic with unit round off $u \approx 1.1 \times 10^{-16}$ ).

The values in columns separated by commas correspond to the solution for derived relations with different orders. This means that in the column for $\mathbf{X}_{l}^{1}$ values 77.3751539 ; $65.8192372 ;-96.0730198 ; 82.9370773$; etc. correspond to $\mathbf{X}_{1}^{1}=77.3751539 ; \quad \mathbf{X}_{2}^{1}=65.8192372 ; \quad \mathbf{X}_{3}^{1}=-96.0730198 ;$ $\mathbf{X}_{4}^{1}=82.9370773$, respectively. The difference in the obtained values (Table I) and results obtained with GEPP algorithm was equal to $1 \times 10^{-14}$. The derived relations produce solutions that are practically identical to the procedure that is most commonly used in solving systems of linear equations.

\section{Proposed Reconstruction Algorithm And UNCERTAINTY ANALYSIS}

The proposed algorithm for signal reconstruction is presented in the form of a flow-chart in Fig. 1.

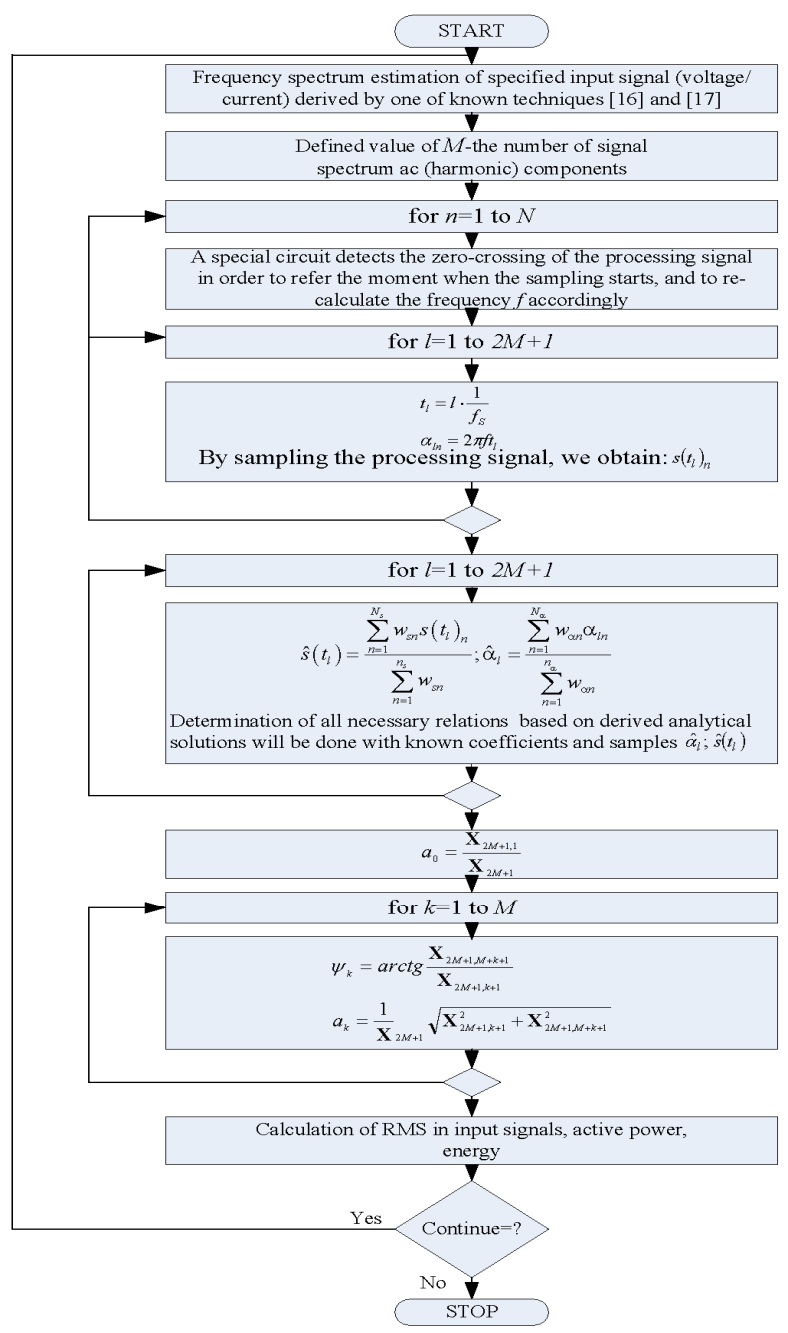

Fig. 1. Flow-chart of proposed reconstruction algorithm.

TABLE I: RESULTS OBTAINED THROUGH THE APPLICATION OF THE DERIVED EXPRESSIONS (7) AND (11)-(13)

\begin{tabular}{cl}
\hline \hline $\mathbf{X}_{2 M+1}$ & \multicolumn{1}{c}{$\mathbf{X}_{l}^{1} ; l=1, \ldots, 15$} \\
\hline & $77.3751539 ; 240.7369977 ;$ \\
$585.7387744 ; 1048.6023899 ;$ \\
$1601.6571515 ; 2122.6636989 ;$ \\
$2515.8984526 ; 2655.5007221 ;$ \\
$557.8121810 ; 2190.4815359 ;$ \\
$1686.1064501 ; 1129.5098525 ;$ \\
64.2746562 & $94.0058063 ; 279.7307319 ;$ \\
& \\
\hline
\end{tabular}

When using the proposed algorithm, the first step to be taken is to ensure that the order of the highest $M$ harmonic component in the processed signal spectrum is known in advance or adopted in advance, accepting that an $M$ determined in this way is bigger than the expected (real) value. One of the well-known methods can be used to estimate the frequency spectrum. Two accurate frequency 
estimation algorithms for multiple real sinusoids in white noise based on the linear prediction approach have been developed in [12]. The first algorithm minimizes the weighted least squares (WLS) cost function, subject to a generalized unit-norm constraint. At the same time, the second method is a WLS estimator with a monic constraint. Both algorithms give very close frequency estimates whose accuracies attain Cramér-Rao lower bound for white Gaussian noise. A modified parameter estimator based on a magnitude phase-locked loop principle was proposed in [13]. It showed that the modified algorithm provided tracking improvements for situations in which the fundamental component of the signal became small, or disappeared for certain periods of time.

In order to recalculate unknown parameters (amplitude and phase) of the processed periodic signals, it is necessary to have the results of the sampling of the input analogue signals $s\left(t_{\nu}\right)$, (equation (2)). The sampling of the input signal must be done in $2 M+1$ points, so as to be able to re-calculate all of the unknown values. The samples of the input signal are obtained by means of sampling in a precisely defined time moments, which are referred in relation to the detected moment of zero crossing. The values of the derived expressions depend on the measured frequency $f$ (Fig. 2), because the values of the determinant elements are calculated based on coefficient $\alpha_{l}$, according to equation (4). Apart from this, other parameters of the derived system of equations will not be dependent on the frequency of the carrier signal and the starting moment of integration of the input signal, as was the case in [7].

Due to the presence of the error in determining the samples $s\left(t_{l}\right)$ and variables $\alpha_{l}$, which is caused by their dependence on the carrier frequency $f$ of the processed signal, in the practical applications of the proposed algorithm we need to have the best estimate of the given values, according to the criterion assumed. This can be done by the means of recalculation of the values $s\left(t_{l}\right)$ and $\alpha_{l}$, through $N$ passages, ( $N$ is arbitrary). In this process we form series $s\left(t_{l}\right)_{n}$ and $\alpha_{l n}(n=1, \ldots, N)$, as it is given in the proposed algorithm (Fig. 1). The random errors $\Delta_{n}$ of measurements are unbiased, $E\left(\Delta_{n}\right)=0$ have the same variance $\operatorname{var}\left(\Delta_{n}\right)=\sigma^{2}$, and are not mutually correlated. Under these assumptions, we can use the weighted average procedure for decreasing random errors in determination of observed values. The weighted average is used for measurements that are not correlated and have a varying degree of accuracy. The averages $\hat{s}\left(t_{l}\right), \hat{\alpha}_{l}$ of the values $s\left(t_{l}\right)$ and $\alpha_{l}$ are calculated for all $l=1,2, \ldots, 2 M+1$ and $n=1,2, \ldots, N$ as:

$$
\hat{s}\left(t_{l}\right)=\frac{\sum_{n=1}^{N_{s}} w_{s n} s\left(t_{l}\right)_{n}}{\sum_{n=1}^{n_{s}} w_{s n}} ; \hat{\alpha}_{l}=\frac{\sum_{n=1}^{N_{\alpha}} w_{\alpha n} \alpha_{l n}}{\sum_{n=1}^{n_{\alpha}} w_{\alpha n}} ; \sum_{n=1}^{N_{s}} w_{s n}=\sum_{n=1}^{N_{\alpha}} w_{\alpha n}=N
$$

where $w_{s n}, w_{\alpha n}$, are non-negative weights of series $s\left(t_{l}\right), \alpha_{l}$. The $N_{s}, N_{\alpha}$, defines the numbers of different values in above series through $N$ passages. The same result (equation (15)), given the adopted suppositions, will be produced by the last square (LS) estimator, after performing the minimisation of the residual sum of squares. The value of $N$ will depend on the required speed of processing - the higher the $N$, the more precise the estimation of the value is. In the concrete case, the estimation procedure does not require the matrix inversion and is considerably less demanding from the processor aspect. In addition to this, when the proposed algorithm is used in simulations, the estimation of the given variables (equation (15)) will not be necessary, which will significantly reduce the processing time needed for its realization. The proposed solution can be modified, in order to reduce the error in determining the samples of the input signal. In [14] it was shown that implementation of sampling and reconstruction with internal antialiasing filtering radically improves performances of digital receivers, enabling reconstruction with a much lower error.

It is necessary to note that the frequencies of the proposed signal may show some differences in relation to the given ones - that is to say that a large or a small frequency mismatch (FM) may exist in real applications. In [15], [16], a new least mean squares (LMS) based Fourier analyser is proposed. This analyser works simultaneously - on one side, it estimates the discrete Fourier coefficients (DFCs) and on the other side, it accommodates the FM. This analyzer can very well compensate for the performance degeneration due to the FM. With every passage of the described algorithm (Fig. 1), the moment of sampling is referred to the detected zero-crossing of the processed signal, and its basic frequency is also calculated at the same time. In this way, the determination of the unknown parameters of the processed periodic signal is less dependent on the possible FM, when compared to the algorithm analyzed in [15], [16] (the parameters analyzed in this paper are less inter-dependent). However, if the signal-to-noise ratio were to be very low and accompanied by a marked FM, it would still be possible to modify the described estimation procedure in a way presented in [15], [16], without adding any requirements to the realization.

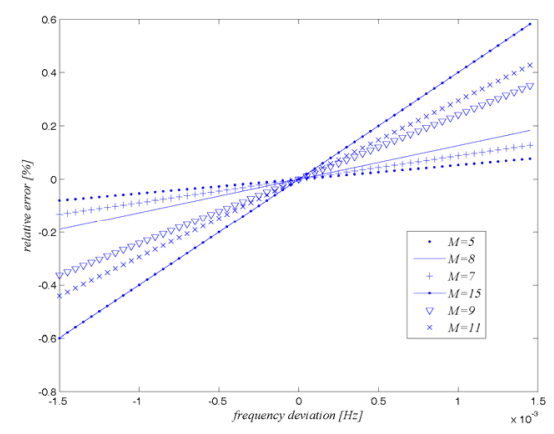

Fig. 2. Relative error in calculation of the system determinant as function of error in synchronization with frequency of fundamental harmonic of the input signal $\left(f=50 \mathrm{~Hz}, t_{l}=0.001 \mathrm{~s}\right)$

After using the procedure described above to perform the estimation of the value of samples $s\left(t_{l}\right)$ and variables $\alpha_{l}$, it is necessary to perform the re-calculation of the unknown amplitudes and phases, based on the relation (14), and for all of the harmonic components of the processed band-limited periodic signal. According to the Fourier coefficients determined in this way, it is possible to perform the calculation of the effective value of the signal, the active power and energy. When this is done, it is possible to start again the sampling of the processed signal.

Fig. 2 shows the influence of the error in determining the 
frequency of the carrier signal on the relative error in determining the value of system determinant, for various harmonic contents of the input periodic signal. It is this very type of error that has the greatest impact on the calculated value of determinants and co-determinants (equations (7) and (11)-(13)), due to the manner in which the values of the elements in these determinants are defined (equation (4)). The immunity of algorithm could be improved by applying a more complex algorithm for the detection of signal zero-crossing moments [17]. The error that appears because of the supposed non-idealities that exist in the suggested reconstruction model is within the boundaries specified by [18]. A sensitivity function is commonly formulated assuming noise-free data. This function provides point-wise information about the reliability of the reconstructed signal before the actual samples of the signal are taken. In [18], the minimum error bound of signal reconstruction is derived assuming noise data.

Unlike the procedure described in [7], the algorithm proposed here is much less sensitive to the variation in the frequency of the carrier signal. The moments $t_{l}$ in which the sampling of the input signal is done can be completely random (asynchronous) and independent of the frequency of the processed signal, due to the way in which they are defined in equation (4). The interval between two consecutive samples is actually dependent primarily on the speed of the $\mathrm{S} / \mathrm{H}$ (sample and hold) circuit and the AD (analogue to digital) conversion circuit, with which a numeric equivalent to the sample of the input signal is formed. However, due to the practical realization and the way in which real sigma-delta ADC function, the $t_{l}$ moments can be defined as $t_{l}=l t_{\text {sample }}$, where $t_{\text {sample }}=1 / f_{S}$ ( $f_{S}$ is the sampling frequency). The start of the sampling must be referred in relation to the detected zero crossing of the input signal.

\section{A. Numerical Complexity of Proposed Algorithm}

Based on the type of the derived relations ((7) and (11)-(13)), the conclusion is that their realization requires a total of $(2 M+1)\left(4 M^{3}+2 M+1\right)$ multiplications and $(2 M+1)\left(2^{2 M}-\frac{1}{2}\left(\begin{array}{c}2 M \\ M\end{array}\right)\right)$ additions. However, due to the method used to determine the unknown parameters of the processed signals (equation (14) and Fig.1); the necessary number of numeric operations is significantly reduced. When the form of the derived relations (11)-(13) is observed, it can be noticed that they contain products that are almost completely identical. In other words, only the products in the denominators of the derived relations have a slightly different form. After the calculation defined in (14), the common factors in the formed products are abbreviated during the division operations. For this reason, the total number of operations is reduced to $18 M^{2}+12 M+2$, meaning that the proposed algorithm required $\frac{9}{2}(2 M+1)^{2}$ flops. This number of operation counts was obtained for the case involving $\mathrm{N}=1$ (without an estimation procedure, the equation (15)), which requires the additional $(2 N+2)(2 M+1)$ multiplications and $4 N(2 M+1)$ additions to be realized. If, in addition to this, the described estimation procedure is performed, then the proposed algorithm takes only $O\left(N(2 M+1)+(2 M+1)^{2}\right)$ flops.

The analytical expressions (11)-(13) are correct, while the error that occurs in their implementation appears because of the numeric procedure used in their calculation (in realizing adding and multiplication). The values of the possible error are defined and analyzed in [19], while it was also proved that the algorithms that solve the Vandermond-like systems (equation (3)) are much more accurate (but no more backward stable) than GEPP (which requires $\frac{2}{3}(2 M+1)^{3}$ flops) or algorithm with QR factorization (which requires $\frac{4}{3}(2 M+1)^{3}$ flops). It follows that proposed algorithm has a higher efficiency than other well-known methods used for solving system of equations like the systems obtained here (3), [19].

While the computational load of one iterative step involving FFTs (which requires $\mathrm{O}((2 M+1) \log (2 M+1))$ flops) does not change with the number of sampling values for some of the non-matrix implementations, the speed of convergence is improved if more points are available. This also corresponds to the natural intuition that the knowledge of more sampling values should lead to better convergence. A larger number of sampling points or a larger spectrum forces to deal with larger matrices. Therefore the computational load for standard matrix methods (either iterative or those using pseudo-inverse matrices) increases quickly. Thus they may be extremely efficient for the situation with a few sampling points, but fairly slow if there are many sampling points. Quite the contrary happens for the methods proposed here. The suggested algorithm is non-iterative and therefore much faster. Also, only the number of the unknowns defines the number of samples required by the proposed algorithm in order to perform the reconstruction in the observed system (3) $(2 M+1$ unknown is parameters). This is opposed to the FFT, where the precision increases with the increased number of samples. This is the main reason why derived analytical solution is more computationally attractive for moderately sized problems [19]. Moreover, this feature makes it feasible for large reconstruction problems. In addition, the method is free from the effects of spectral leakage, which a common problem in reconstruction algorithm based on the use of FFT.

\section{B. Computing Time}

The suggested algorithm can be applied in operation with sigma-delta ADC, thus enabling high resolution and speed in processing of input signals. This is an important difference to be taken into consideration when comparing its implementation in this approach (to processing), to the results presented in [7]. The time needed to perform the necessary number of samplings of the input signal that is the object of reconstruction can be defined as $(2 M+1) t_{\text {sample }}$, which represents the value approximate to the time needed for reconstruction (in simulation). In practical applications of the proposed algorithm, the determined time for the reconstruction of the processing signal ought to be increased by the time necessary to estimate the variables $s\left(t_{l}\right)$ and $\alpha_{1}$ 
(this time is directly dependent on the value $\mathrm{N}$ ), and the time interval $\Delta \mathrm{t}$ which is necessary to perform all the other re-calculations according to the proposed algorithm. Due to all that has been said, the reconstruction time can be defined as $N(2 M+1) t_{\text {sample }}+\Delta t \approx N \frac{1}{f}+\Delta t$, because of the necessary synchronization with the zero crossing of the input signal. The speed of the proposed algorithm makes it as fast as the algorithms analyzed in [20], [21].

In order to demonstrate the efficiency of the new procedure there is a comparison of the computing time of the proposed algorithm to GEPP algorithm in solving the system of equations (3), Table II (using Matlab program package, version R2006a). The circumstance of verifying real-time characteristic is in computer with Intel Pentium 2.0G Dual CPU, 2Gb RAM, and Windows XP 2002 operation system. The results given in Table II practically present the estimate value of the time interval $\Delta t$. The proposed procedure shortens the time needed for calculation by 2 to 3 times, depending on the number of the harmonic components of the processed signal. With a more powerful hardware platform and a different program environment, the time for the realization of the proposed algorithm will be many times shorter.

Reference [22] gives a measurement of the required processor time, in the realization of the matrix method in the reconstruction of signals, in the form in which it is implemented in many program packages. The method suggested by this paper does not require any special memorization of the transformation matrix, nor does it require recalculation of the inversion matrix. In this way, it is much more efficient in implementation and it is not limited only to sparse matrices.

\section{Simulation of the Proposed ReCONStRUCtion ALGORITHM}

Additional testing of the proposed algorithm was carried out by simulation in the program package Matlab and SIMULINK. Since measurement is corrupted by noise, the reconstruction is an estimation task, i.e. the reconstructed signal may vary, depending on the actual noise record. We investigate the issue of noise and jitter on the estimation method. The presence of the noise and jitter causes false detection of signal zero-crossing moments, giving an incorrect calculation of derived relations. Apart from this, an error occurs in determining the value of the samples of the processed signal. This error appears as a result of the imprecision in determining the time moment at which sampling is done, as well as a result of the error introduced by the real ADC.

Since the suggested reconstruction algorithm is practically based on the standard hardware components with which the sampling of the input analogue signal is done, the model that was used in simulation was the system shown in Fig. 3. A sigma-delta ADC with corresponding circuit for $\mathrm{S} / \mathrm{H}$ was used to perform the conversion [23]; at the same time, the measuring of the frequency of the carrier signal was done in the way suggested in [17], i.e. by using comparator
(Schmitt-triggers), we can detect the passing of the multi-harmonic input signal through zero and in this way detect this frequency.

TABLE II: COMPARISON OF THE COMPUTING TIME

\begin{tabular}{lll}
\hline \hline $\begin{array}{c}\text { Number of } \\
\text { harmonic } \\
\text { components, } M\end{array}$ & PROPOSED ALGORITHM & GEPP algorithm \\
\hline 5 & $0.00087 \mathrm{~s}$ & $0.00171 \mathrm{~s}$ \\
7 & $0.00094 \mathrm{~s}$ & $0.00215 \mathrm{~s}$ \\
8 & $0.00098 \mathrm{~s}$ & $0.00251 \mathrm{~s}$ \\
9 & $0.00106 \mathrm{~s}$ & $0.00297 \mathrm{~s}$ \\
11 & $0.00121 \mathrm{~s}$ & $0.00399 \mathrm{~s}$ \\
15 & $0.00188 \mathrm{~s}$ & $0.00564 \mathrm{~s}$ \\
\hline \hline
\end{tabular}

The input signal is formed as a super-position of harmonic components within the „Multi-harmonic input signal“ block. Since the presence of the noise and jitter causes false detection of signal zero-crossing moments, there is a separate model that simulates their impact on the signal that is processed according to the proposed algorithm. All Taylor expansions of jitter are biased [24]; therefore, the correct way to include jitter is to include a variable delay in the signal path. The model by which the jitter presence is simulated is made of the Pulse Generator and Random Number, whose outputs are taken to the circuit with the Variable Transport Delay. Fig. 3 shows the addition of the white Gauss noise to the complex periodic signal. In the original toolbox all, the possible noise sources (mainly the contributions of the operational amplifiers and of the voltage references) were supposed to be white. The noise power in the block ,Band-Limited White Noise" is the height of the PSD (Power Spectral Density), expressed in $\mathrm{V}^{2} / \mathrm{Hz}$. By using the simple Relay block shown in Fig. 3, the signal formed in this way, is transformed into a series of right-angle impulses, by the means of which the frequency of the fundamental harmonic was measured within the ,S-Function“ block [17].

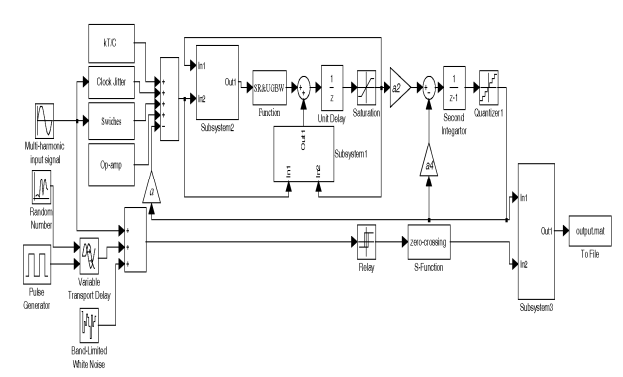

Fig. 3. SIMULINK model of the circuit for the realization of proposed reconstruction algorithm.

\section{A. Model of Sigma-Delta ADC}

In order to have a highly accurate simulation of the conversion circuit, a sigma-delta ADC model described in [25] was used (the effective resolution of the ADC during the simulation was $24 \mathrm{bit}$, and sampling rate $f_{S}=1 \mathrm{kHz}$ ). In order to have a better picture of the performances of the simulation model, Fig. 4 shows detailed models of the clock jitter noise, $k T / \mathrm{C}$ noise and the op-amp's thermal and flicker noise at low frequencies. Considering that the noise power is additive, the PSD can be considered as the sum of a term due to flicker $(1 / f)$ noise and one due to thermal noise, associated to the sampling switches and the intrinsic noise of the operation 
amplifier. Here, $k, T$, and C are Boltzmann's constant, the temperature in Kelvin, and the sampling capacitor, respectively. The $V_{n}$ denotes the input-referred thermal noise of the op-amp. Flicker $(1 / f)$ noise, wide-band thermal noise and dc offset contribute to this value. The total noise power $V_{n}^{2}$ can be evaluated, through transistor level simulation, and during the simulation it is assumed that $V_{n}=30 \mu \mathrm{V}_{\text {rms }}$, while the value of the sampling capacitance was $C=2 \mathrm{pF}$. The effect of clock jitter was simulated by using a model separately shown in Fig. 4, under the assumption that the time jitter is an uncorrelated Gaussian random process having a standard deviation $\Delta \tau$. This model of the jitter was described and analyzed in detail in [26], where it was shown that the noise floor is dependent on the input sinusoidal frequency. During the simulation, the value of jitter was $1 \mathrm{~ns}$ (standard deviation $\Delta \tau)$. In the course of the simulation conducted in this way, the output PSD of the ideal, thermal noise affected and clock jitter affected was in the range of -100 to $-170 \mathrm{~dB}$ for the signal-to-noise distortion ratio (SNDR) ranged between 85 $\mathrm{dB}$ and $96 \mathrm{~dB}$.

The block „Integrator DC Gain“models the finite dc-gain (DCG). The finite DCG moves the pole of the ideal integrator from dc to another frequency and changes the integrator's gain. This effect is known as a leakage in the integrator (a separate block in the developed model). Distortion is introduced by the integrator's DCG nonlinearity resulting from its dependency on the output voltage as shown in Fig. 4. Slew rate (SR) and unity-gain bandwidth (UGBW) are the two distinct parts of the settling behavior of the op-amps, for which reason these are modelled separately. For an integrator, in presence of its op-amp's UGBW and SR, its settling behavior will be linearly/nonlinearly affected [25]. The values of the coefficients of the amplifier circuits in Fig. 4 are $a=1 ; a_{2}=0.5 ; a_{4}=0.25$.

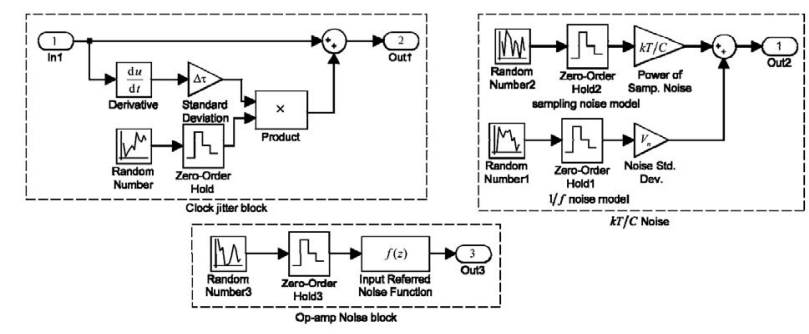

Fig. 4. SIMULINK models of clock jitter, $k T / \mathrm{C}$ and flicker noise, and op-amp noise

Switches are one of the major elements in switched-capacitor (SC) circuits such as the sigma-delta ADC. Since the switches in CMOS technology are realized by using nMOS and pMOS transistor, they manifest some no idealities such as nonlinear on-resistance, clock-feed through, and charge injection (decreasing the $\mathrm{S} / \mathrm{H}$ time constant) [25]. The block „Switches charge-injection“ comprise all of the listed non-idealities.

A quantizer circuit is introduced in the ADC model, using a special block. The quantization error is a very important problem because the reconstruction algorithm proposed here is of a quite sophisticated form and some operations, like determinant calculation for example, are badly conditioned task and may considerably amplify the quantization errors.

\section{B. Simulation Results}

After the values of the input signals samples were determined through the above described ADC model, these were introduced in the „Subsystem3“ block (Fig. 3), together with the information about the measured frequency of the input signal, so that the values of the unknown amplitudes and phases of the input periodic signal can be established, based on the derived relations. During the simulation, the parameters of the input signal correspond to the values given in Table III. The execution time of the proposed algorithm on hardware platform described earlier was $0.0167 \mathrm{~s}$.

A signal containing the first 7 harmonics was used, with the fundamental frequency $f=50 \mathrm{~Hz}$. The superposed noise and jitter will, in simulation performed in this way, cause a relative error in detection on fundamental frequency of $0.0001 \%$. It can be seen that the accuracy of the proposed algorithm is within the limits that are attained in processing a signal of this form, in [21], [27], and better then the one presented in [28]. In the time domain, the relative error between the signal and its reconstruction was $0.0025 \%$. The errors in the amplitude and phase detection are mainly due to the error in measuring of the input signal samples and the error in determining the value of the derived equations.

The presented algorithm can be used for the spectral analysis as well, where it is possible to find out the amplitude and the phase values of the signal harmonic, based on the set (predicted) system of equations. By taking a systematic approach in conducting the described procedure, it is possible to establish the exact spectral content and, after this has been done, to perform the optimization of the proposed algorithm. With this, the algorithm will be adapted to the real form of the signal. The accuracy of signal reconstruction can be guaranteed in practical applications in noisy environments with the use of a powerful processor with adequate filtering.

\section{V.CONCLUSION}

The estimation procedure proposed in this paper is a new complexity-reduced algorithm for estimation of the Fourier coefficient. The derived analytical expression opens a possibility to perform fast calculations of the basic parameters of signals (the phase and the amplitude), with a low numeric error. The proposed algorithm for the calculation of the unknown parameters requires $\mathrm{O}\left((2 M+1)^{2}\right)$ flops, while the computing time is determined by the time that is necessary for the collection of the required number of samples of the processed signal and the estimation procedure itself. In any case, the time is much shorter than with any other known matrix methods used. All the necessary hardware resources can be satisfied by a DSP of standard features and real sigma-delta ADC. The suggested concept can be used as a separate algorithm as well, for the spectral analysis of the processed signals. Based on the identified parameters of the ac signals, we can establish all the relevant values in the electric utilities (energy, power, rms values). The measurement uncertainty is a function of the error in synchronization with fundamental frequency of processing signal (because of the no stationary nature of the jitter-related noise and white Gaussian noise), and the error that occurs in determining the values of the samples of the processed signal. 
The simulation results show that the proposed algorithm can offer satisfactory precision in reconstruction of periodic signals in a real environment.

TABLE III: COMPARISON OF SIMULATION RESULTS BY THE PROPOSED RECONSTRUCTION ALGORITHM, FFT AND CONTINUOUS WAVELET TRANSFORMATION (CWT) [28]

\begin{tabular}{|c|c|c|c|c|}
\hline \multirow{2}{*}{$\begin{array}{l}\text { Harmonic } \\
\text { number }\end{array}$} & \multirow{2}{*}{$\begin{array}{l}\text { Amplitude } \\
\text { [VPP] }\end{array}$} & \multirow{2}{*}{$\begin{array}{l}\text { Phase } \\
\text { [rad] }\end{array}$} & \multicolumn{2}{|c|}{$\begin{array}{c}\text { Proposed reconstruction } \\
\text { algorithm }\end{array}$} \\
\hline & & & $\begin{array}{c}\text { Amp.error } \\
{[\%]}\end{array}$ & $\begin{array}{c}\text { Phase error } \\
{[\%]}\end{array}$ \\
\hline 1 & 1 & $\pi$ & 0.0016 & 0.0021 \\
\hline 2 & 0.73 & $\pi / 3$ & 0.0023 & 0.0018 \\
\hline 3 & 0.64 & 0 & 0.0021 & 0.0022 \\
\hline 4 & 0.55 & $\pi / 6$ & 0.0017 & 0.0019 \\
\hline 5 & 0.32 & $\pi / 4$ & 0.0018 & 0.0024 \\
\hline 6 & 0.27 & $\pi / 12$ & 0.0023 & 0.0017 \\
\hline 7 & 0.14 & 0 & 0.0024 & 0.0023 \\
\hline \multirow[b]{2}{*}{$\begin{array}{c}\text { Harmonic } \\
\text { number }\end{array}$} & \multicolumn{2}{|c|}{$\begin{array}{l}\text { FFT (sampling rate }=25 \\
\mathrm{kHz} ; \\
\text { data length }=25000 ; \text { time } \\
\text { period }=1 \mathrm{~s})\end{array}$} & \multicolumn{2}{|c|}{ CWT } \\
\hline & $\begin{array}{c}\text { Amp.error } \\
{[\%]}\end{array}$ & $\begin{array}{c}\text { Phase } \\
\text { error [\%] }\end{array}$ & $\begin{array}{c}\text { Amp.error } \\
{[\%]}\end{array}$ & $\begin{array}{c}\text { Phase error } \\
{[\%]}\end{array}$ \\
\hline 1 & 0.296 & 0.322 & 0.023 & 0.034 \\
\hline 2 & 0.035 & 0.038 & 0.032 & 0.028 \\
\hline 3 & 0.875 & 0.843 & 0.049 & 0.026 \\
\hline 4 & 0 & 0 & 0.144 & 0.012 \\
\hline 5 & 0 & 0 & 0.013 & 0.154 \\
\hline 6 & 0 & 0 & 0.012 & 0.017 \\
\hline 7 & 0 & 0 & 0.223 & 0.186 \\
\hline
\end{tabular}

\section{ACKNOWLEDGMENT}

The author wishes to thank to the Ministry of Education and Science of the Republic of Serbia for its support of this work provided within the projects 42009 and OI-172057.

\section{REFERENCES}

[1] J. G. Proakis and D. G. Manolakis, Digital Signal Processing. Principles, Algorithms, Applications, 3rd ed. Englewood Cliffs, NJ: Prentice-Hall, 1996.

[2] R. S. Prendergast, B. C. Levy, and P. J. Hurst, Reconstruction of Band-Limited Periodic Nonuniformly Sampled Signals Through Multirate Filter Banks," IEEE Trans. Circ. Syst.-I, vol. 51, no. 8, pp.1612-1622, Aug. 2004.

[3] P. Marziliano, M. Vetterli, and T. Blu, Sampling and Exact Reconstruction of Bandlimited Signals with Additive Shot Noise," IEEE Trans. Inform. Theory, vol. 52, no. 5, pp.2230-2233, May 2006.

[4] E. Margolis and Y. C. Eldar, Reconstruction of nonuniformly sampled periodic signals: algorithms and stability analysis," Electronics, Circuits and Systems, 2004. ICECS 2004. Proceedings of the 2004 11th IEEE International Conference, 2004, pp. 555-558.

[5] W. Sun and X. Zhou, Reconstruction of Band-Limited Signals From Local Averages," IEEE Trans. Inf. Theory, vol. 48, no. 11, pp. 2955-2963, Nov. 2002

[6] A. K. Muciek, A Method for Precise RMS Measurements of Periodic Signals by Reconstruction Technique with Correction," IEEE Trans. Instrum. Meas, vol. 56, no. 2, pp.513-516, Apr. 2007.

[7] P. Petrovic, "New approach to reconstruction of nonuniformly sampled AC signals," Proceedings of 2007 IEEE International Symposium on Industrial Electronics (ISIE 2007), Vigo, Spain, 2007, pp. 1693-1698.

[8] Y. Xiao, Y. Tadokaro, and K. Shida, Adaptive Algorithm Based on Least Mean p-Power Error Criteterion for Fourier Analysis in Additive Noise," IEEE Trans. Signal Proc., vol. 47, no. 4, pp.1172-1181, Apr. 1999.
[9] M. Agrawal, M, S. Prasad S, and S. C. D. Roy, "A simple solution for the analytic inversion of Van der Monde and Confluent Van der Monde matrices," IETE JOURNAL OF RESEARCH, vol. 47, no. 5, pp. 217-219, Sep.-Oct. 2001

[10] I. Gohberg and V. Olshevsky, The fast generalized Parker-Traub algorithm for inversion of Van der Monde and related matrices," $J$. Complexity, vol. 13, no.2, pp. 208-234, June 1997.

[11] C. S. Jog, On the explicit determination of the polar decomposition in n-dimensional vector spaces", JOURNAL OF ELASTICITY, vol.66, no. 2, pp.159-169, Dec. 2002.

[12] H. C. So, K. W. Chan, Y. T. Chan, and K. C. Ho, Linear Prediction Approach for Efficient Frequency Estimation of Multiple Real Sinusoids: Algorithms and Analyses", IEEE Trans. Signal Proc, vol. 53, no. 7, pp.2290-2305, July 2005.

[13] B. Wu and M. Bodson, "Frequency estimation using multiple source and multiple harmonic components," American Control Conference, 2002. Proceedings of the 2002, vol.1, 2002, pp. 21-22.

[14] Y. S. Poberezhskiy and G. Y. Poberezhskiy, Sampling and Signal Reconstruction Circuits Performing Internal Antialiasing Filtering and Their Influence on the Design of Digital Receivers and Transmitters,' IEEE Trans. Circ. Sys.-I, vol. 51, no. 1, pp. 118-129, Jan. 2004.

[15] Y. Xiao, R. K. Ward, L. Ma, and A. Ikuta, "A New LMS-Based Fourier Analyzer in the Presence of Frequency Mismatch and Applications," IEEE Trans. Circ. Syst.-I, vol. 52, no. 1, 2005, pp.230-245, Jan. 2005

[16] Y. Xiao, R. K. Ward, and L. Xu, "A new LMS-based Fourier analyzer in the presence of frequency mismatch," ISCAS'03, Proceedings of the 2003 International Symposium on Circuits and Systems, vol. 4, 2003, pp.369-372.

[17] P. Petrovic, New Digital Multimeter for Accurace Measurement of Synchronously Sampled AC Signals," IEEE Trans. Instrum. Meas, vol. 53, no.3, 2004, pp.716-725, June 2004.

[18] G. Wang and W. Han, "Minimum Error Bound of Signal Reconstruction," IEEE Signal Proc. Lett., vol. 6, no. 12, pp. 309-311, Dec. 1999

[19] N. J. Nigham, Accuracy and Stability of Numerical Algorithms, 2nd ed, SIAM, 2002.

[20] T. Cooklev, „An Efficient Architecture for Orthogonal Wavlet Transforms“, IEEE Signal Proc. Lett. vol. 13, no. 2, pp. 77-79, Feb. 2006.

[21] J. Schoukens, Y. Rolain, G. Simon, and R. Pintelon, Fully Automated Spectral Analysis of Periodic Signals, IEEE Trans. Instrum. Meas. vol. 52, no. 4, pp.1021-1024, Aug. 2003.

[22] S. J. Reeves, An Efficient Implementation of the Backward Greedy Algorithm for Sparse Signal Reconstruction," IEEE Signal Proc. Lett. vol. 6, no. 10, pp. 266-268, Oct. 1999.

[23] P. Petrovic, New procedure for estimation of amplitude and phase of compelx ac signals", in Proc. I2MTC, Austin, TX, USA, 2010, pp.464-469.

[24] K. J. Coakley, C. M. Wang, P. D. Hale, and T. S. Clement, Adaptive characterization of jitter noise in sampled high-speed signals," IEEE Trans. Instrum. Meas. vol. 52, no. 5, pp.1537-1547, Oct. 2003.

[25] H. Z. Hoseini, I. Kale, and O. Shoaei, Modeling of Switched-Capacitor Delta-Sigma Modulators in SIMULINK“, IEEE Trans. Instrum. Meas. vol. 54, no. 4, pp.1646-1654, Aug. 2005.

[26] G. Vendersteen and R. Pintelon, Maximum likelihood estimator for jitter noise models“, IEEE Trans. Instrum. Meas. vol. 49, no. 6, pp.1282-1284, Dec. 2000.

[27] R. M. Hidalgo, J. G. Fernandez, R. R. Rivera, and H. A. Larrondo, A Simple Adjustable Window Algorithm to Improve FFT Measurements," IEEE Trans. Instrum. Meas. vol. 51, no. 1, pp.31-36, Jan. 1996

[28] N. C. F. Tse and L. L. Lai, "Wavelet-Based Algorithm for Signal Analysis", EURASIP Journal on Advances in Signal Processing, vol. 2007, Article ID 38916, 10 pages, 2007.

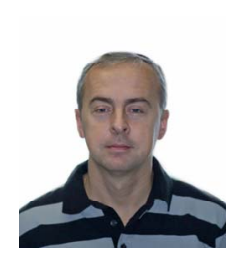

Predrag B. Petrović was born in Čačak, Yugoslavia, on January 26, 1967. He received his BS and MS degrees in electrical engineering from the University of Belgrade, Yugoslavia in 1991 and 1994, respectively, and his $\mathrm{PhD}$ degree in Digital Signal Processing from the University of Novi Sad in 2004. Since 2011, he held a position as Full Professor at the University of Kragujevac, Čačak College of Engineering. His main interests are digital signal processing, microcontroller programming, power electronics, $\mathrm{AD}$ conversion, mathematics, and cryptology. He has published more than 100 journal and conference papers, five university books, one international monograph and holds four patents. 